\title{
O lema Kuratowski-Zorn na matemática do Ensino Médio
}

\author{
Kuratowski-Zorn lemma in High Scholl mathematics
}

EI lema Kuratowski-Zorn em matemáticas de la Escuela Secundaria

Recebido: 30/01/2021 | Revisado: 04/02/2021 | Aceito: 31/03/2021 | Publicado: 09/04/2021
Eduardo da Conceição Rosário
ORCID: https://orcid.org/0000-0001-9435-2522
Instituto Federal de Educação, Ciência e Tecnologia do Amapá, Brasil
E-mail: eduardo.rosario@ ifap.edu.br
Cassio Alessandro Batista Balieiro
ORCID: https://orcid.org/0000-0003-2380-9163
Universidade Federal do Amapá, Brasil
E-mail: balieiromatematico@ gmail.com
Neylan Leal Dias
ORCID: https://orcid.org/0000-0001-9659-8825
Universidade Federal do Amapá, Brasil
E-mail: neylanmatematico@ gmail.com
Simone de Almeida Delphim Leal
ORCID: https://orcid.org/0000-0002-7346-3159
Universidade Federal do Amapá, Brasil
E-mail: leal@ unifap.br

\begin{abstract}
Resumo
O Lema Kuratowski-Zorn denominado frequentemente apenas por Lema de Zorn é objeto de controvérsias e debates desde o seu surgimento, apesar disto, sua relevância pode ser percebida pela grande quantidade de aplicações e consequências apresentadas sobre a forma de enunciados equivalentes que são presentes na área de exatas, e também em aplicações matemáticas que se baseiam na teoria dos conjuntos como, axioma da Escolha, Teorema da Boa ordem, Teorema de Tychonoff, Teorema Hahn Banach entre outros, sendo os três primeiros equivalentes ao Lema de Zorn. Por este motivo, teremos como objetivo, partindo de uma abordagem histórica sobre o mesmo, apresentar umas das aplicações que são responsáveis por fortalecer sua notoriedade e popularidade entre estudiosos matemáticos e cientistas das mais variadas áreas que se utilizam da Álgebra Linear para representar e resolver seus modelos matemáticas utilizando este Lema e suas equivalências, onde veremos como podemos encontrá-lo na matemática do ensino médio regular das escolas quando apresentada aos alunos.
\end{abstract}

Palavras-chave: Lema de Zorn; Teoria dos conjuntos; Álgebra linear; Axioma de escolha.

\begin{abstract}
Zorn's lemma has been the subject of controversy and debate since its emergence, despite its relevance can be perceived by the large number of applications and consequences presented on the form of equivalent statements that are present in the area of exact, and also in mathematical applications that they are based on the set theory as, axiom of Choice, Theorem of Good Order, Tychonoff's Theorem, Hahn Banach Theorem among others, the first three being equivalent to Zorn's Lemma. For this reason, we will aim, starting from a historical approach on the same, to present one of the applications that are responsible for strengthening its notoriety and popularity among mathematical scholars and scientists from the most varied areas that use Linear Algebra to represent and solve their problems. mathematical models using this lemma and its equivalences, where we will see how we can find it in the mathematics of regular high school schools when presented to students.
\end{abstract}

Keywords: Zorn's lemma; Set theory; Linear algebra; Axiom of choice.

\section{Resumen}

El lema Kuratowski-Zorn ha sido objeto de controversia y debate desde sus inicios, a pesar de su relevancia se puede percibir por la gran cantidad de aplicaciones y consecuencias que se presentan en forma de enunciados equivalentes que están presentes en el área de los exactos, y también en los matemáticos. aplicaciones que se basan en la teoría de conjuntos como, axioma de elección, teorema del buen orden, teorema de Tychonoff, teorema de Hahn Banach entre otros, siendo los tres primeros equivalentes al lema de Zorn. Por ello, nos proponemos, partiendo de un enfoque histórico sobre el mismo, presentar una de las aplicaciones que se encargan de fortalecer su notoriedad y popularidad entre los estudiosos matemáticos y científicos de las más variadas áreas que utilizan el Álgebra Lineal para representar y resolver. sus problemas modelos matemáticos utilizando este lema y sus equivalencias, donde veremos cómo podemos encontrarlo en las matemáticas de las escuelas secundarias regulares cuando se presentan a los estudiantes. 
Palabras clave: Kuratowski-Zorn; Teoría de conjuntos; Álgebra lineal; Axioma de elección.

\section{Introdução}

Considerando a relevância de estudos que apontam o uso da história matemática como um importante recurso capaz de proporcionar uma conexão entre a teoria e a prática (Pereira \& Saito, 2018), e uma vez que hoje muitas pessoas estudam os lemas, axiomas e teoremas sem necessariamente saber de sua história ou de como chegou a tal nome, iniciaremos este trabalho esclarecendo um mistério que cerca a origem de um dos Lemas mais conhecidos no mundo matemático. O lema de Zorn que leva consigo o nome de Max Augustin Zorn, que em 1935 o apresentou em um trabalho sobre princípios maximais. Mais tarde, uma carta abalou a sociedade Matemática das Américas, pois a mesma trazia a notícia de que o Lema da Zorn não possuía o nome de seu verdadeiro descobridor. Nesta busca pelas origens do lema de Zorn, foram encontrados documentos que comprovam, além de contribuição de diversos autores, o seu uso anterior as pesquisas de Zorn (Campbell, 1978).

Segundo (Campbell, 1978), o lema conhecido atualmente como" Lema de Zorn” já havia sido citado por outros autores, como: (Rosser, 1953, Cuesta, 1955, Fraenkel \& Bar-Hillel, 1958, Rubin \& Rubin, 1963, Beth, 1964, Semadeni, 1968, Jech 1973 \& Suppers, 1960). Muitos desses autores acabaram por estudar o tal Lema de Zorn, a história escondida por trás do nome que chama atenção, pois Max já conhecia relatos de outros estudos sobre o Lema.

Esses supostos trabalhos faziam referências ou são equivalentes ao Lema de Zorn conhecido hoje, alguns desses trabalhos eram vistos e revistos por seus autores, pois muitos possuíam equívocos e tinham que ser reajustados. Alguns autores faziam, por vezes, referências a resultados de outros autores para elaborar definições ou reformular. Campbell relata uma forma de linhagem de como o princípio maximal tornou-se lema de Zorn, chegando a conclusão de que certamente Zorn foi essencialmente antecipado por alguns autores. Acontecimentos esses que passamos a descrever.

Max Zorn, era Professor de uma universidade de Indiana e fez um trabalho sobre o princípio do máximo em torno de 1933, como de caso "Principio trabalho" em álgebra. Hausdorff já havia trabalhado com os princípios maximais pois ele foi o primeiro a fazer isso, mas Max só tomou conhecimento de tal trabalho em 1976, apesar disto Hausdorff foi o primeiro a chamar o princípio maximal de Lema de Zorn (passou a ser chamado assim depois de sua apresentação nas américas em outubro de 1934). Sobre o seu trabalho em álgebra, Max apenas se deixou aceitar a terminação Lema de Zorn, apesar de ter contribuído bastante, ele tinha conhecimento das linhagens que o sucederam em alguns resultados. Kuratowski foi o Primeiro a utilizar o Lema de Zorn, ainda hoje podemos ver algumas notações do lema de Zorn, como Lema de Kuratowski-Zorn (Rubin, \& Rubin, 1963).

\section{Metodologia}

A presente pesquisa classificada segundo os meios utilizados é do tipo bibliográfica, por consistir em uma revisão da literatura disponível relativa ao tema abordado. Com este fim, foram utilizadas diversas fontes, dentre elas websites, artigos e livros.

Segundo (Boccato, 2006), "esse tipo de pesquisa é capaz de subsidiar o conhecimento sobre o que foi pesquisado, como e sob que enfoque e/ou perspectivas foi tratado o assunto apresentado na literatura científica". A partir desta concepção, passa-se à descrição da terminologia relacionando-as à suas referências históricas.

Naquela época, alguns autores faziam referências aos seus trabalhos nomeando-os como: M1, M2, M3 que são conhecidos como princípios Maximai. Kuratowski foi o primeiro a demonstrar que o Lema era equivalente ao Axioma da Escolha, S. Bochner fez um artigo sobre superfícies de Riemann, que foi Chamado por Rubin e Rubin de M1, foi estudado por Hausdorff e era tratado com Princípio de Zorn que diz: 
M1: Se R é uma relação transitiva em um conjunto não vazio, que definimos de $\mathrm{X}$ e se todo subconjunto de $\mathrm{X}$ é linearmente ordenado por R e tem uma parte superior de $\mathrm{R}$ ligado a esse subconjunto, então há uma relação $\mathrm{R}$ maximal em um elemento de $\mathrm{X}$.

O trabalho de (Rubin \& Rubin, 1963) foi importante, pois apresenta reformulações do Axioma da Escolha, diferencia vários princípios maximais estudando-os e analisando-os. Alguns dos princípios Maximais são definidos por M3 e M4.

M3: Se todas as classes não vazias são um subconjunto de um não vazio $X$, então $X$ tem em sua união um elemento dele próprio, em seguida $\mathrm{X}$ tem um elemento máximo.

M4: Se cada classe bem-ordenada e um subconjunto de um não vazio $X$, então $\mathrm{X}$ tem em sua união um elemento dele mesmo, logo X tem um elemento máximo.

Onde M3 é o que se assemelha com o lema de Zorn que conhecemos hoje em dia e M4 já apareciam em um teorema topológico de 1910, e sua demonstração foi baseada no teorema de boa ordem por alguns autores como: Janiszewski, Mazurkiewicz, Brouwer e Kuratowski, Rubin.

Rubin e Rubin após demonstrarem que o princípio do máximo implicava no axioma da escolha e no teorema de Boaordem, optaram por não publicar o resultado, então Max Zorn, que chegou a mesma conclusão, aplicou na álgebra o princípio maximal que ficou conhecido como lema de Zorn.

Zorn tomou conhecimento de vários princípios maximais, como os que Hausdorff formulou, provou e estendeu, a conclusão de Rubin e Rubin chamando de M5 e M6, que dizem:

M6: Para cada conjunto X, existe um subconjunto maximal.

M5: Se R é uma relação transitiva em X, então existe um subconjunto maximal com X ordenado por R.

Tomando os conhecimentos de alguns maximais existentes, Zorn formulou duas definições que são:

Definição 1: Um conjunto B de conjuntos B é chamado uma cadeia, se para cada dois conjuntos B1, B2, tal que B1 つ B2 ou $\mathrm{B} 2 \supset \mathrm{B} 1$.

Definição 2: Um conjunto A de conjuntos A é dito fechado, se contiver a união SB de cada cadeia B contido em A. Então, o nosso princípio maximal é expressado no seguinte formato (PM).

Em um sistema fechado, o conjunto $\mathrm{A}$ de conjuntos $\mathrm{A}$ existe pelo menos um, $\mathrm{A}^{*}$ que é o maximal, e não está contido em nenhum subconjunto A.

A partir de então, com os conhecimentos sobre outros princípios maximais, Zorn passou a estudá-los antes de chegar a essas definições, o Lema de Zorn atual diz o seguinte:

Se X é um conjunto parcialmente ordenado tal que para todo subconjunto e totalmente ordenado em X, há uma cota superior em X, então X contém um elemento maximal. Sua demonstração pode ser encontrada em (Halmos, 2001).

Bourbaki fez uma formulação que chamou de Lema Fundamental, afim de discutir sobre o teorema de Zorn, como já eram chamadas as formulações de Max, que diz que: 
Seja $E$ um conjunto ordenado indutivo. Então é uma aplicação de $E$ em $E$, tal que, para todo $x \in E, f(x) \geq x$, existe pelo menos um $x \in E$ tal que $f(x)=x$.

O Lema de Zorn surgiu de uma fusão entre o Teorema de Zorn e Lema Fundamental, de Bourbaki que diz:

Seja $\mathrm{E}$ um conjunto ordenado indutivo, e $\mathrm{F}$ uma aplicação de $\mathrm{E}$ em $\mathrm{E}$, tal que, para todo $\mathrm{x}$ pertence a $\mathrm{E}$, temos que $\mathrm{f}(\mathrm{x})$ $=\mathrm{x}, \log 0$ existe ao menos um elemento $\mathrm{x}$ pertencente a E tal Que $\mathrm{f}(\mathrm{x})=\mathrm{x}$, (Silva \& Jesus, 2007).

Hoje em dia é mais conhecido como Teorema de Zorn, sendo bastante utilizado ainda em análise funcional, topologia, álgebra linear, em especial na teoria dos conjuntos, pois apesar de não ser usado tanto para uma construção axiomática de Conjuntos, sem ele não conseguiríamos demonstrar tão facilmente, o teorema de Hanh-Banach ou que um espaço de Hammel possui uma base.

Considerando que a álgebra linear é uma parte da matemática usada por interessados nas mais diversas ciências exatas como engenharia, física, programação e, ainda que sua importância não se restrinja a área de exatas, atingindo também questões da atualidade como sistemas ligados à biologia, podemos conceber a sua relevância para o desenvolvimento de várias ciências. Essa importância nos conduziu na escolha da aplicação a ser apresentada, que trata de um dos conceitos mais primitivos de espaços vetoriais de dimensão finita.

Quando se trata de aprendizagem dessas disciplinas muitos alunos possuem certas restrições, tratando-as apenas como letras misturadas com números, para uma complicação em seu aprendizado na disciplina. A álgebra vem praticamente desde o início da humanidade e vem sendo aplicada no ensino regular desde de seu surgimento, o que difere da teoria dos conjuntos que, este por sua vez, passou a ser utilizado a partir do ano de 1960 e, muitas das vezes em algumas escolas, é deixada de lado no ensino médio, fazendo com que o aluno fique em uma lacuna difícil de ser suprida em estudos próximos (Celestino, 2000).

A teoria dos conjuntos sofreu um grande preconceito quando adicionada ao ensino regular nas escolas, já que a mesma veio com o objetivo de substituir a geometria na época e seu ensino de aprendizagem repousa no fato de que hoje se é fundamental o seu aprendizado nas escolas e, não apenas da teoria dos conjuntos, mas também da álgebra, já que as duas praticamente caminham juntas apesar de algumas diferenças quando a tratamos mais aprofundamentos (Diniz, 2019).

Hoje em dia devemos nos preocupar de como está sendo abordada a teoria dos conjuntos e a álgebra nas escolas, pois estas são praticamente as bases da matemática, cientificamente falando, e não as deixar de lado, pois isso dificultará o aprendizado do aluno nos demais assuntos a serem compreendidos.

A Teoria dos conjuntos e a Álgebra linear existem desde os primórdios da matemática, seus conceitos tais como funções, relações, espaços vetoriais e produtos cartesianos, são bases de diversos ramos da matemática, além disso, por serem apresentadas como corpos de fatos e de axiomas, muitas das vezes elas são ensinadas por partes, partindo do conceito de conjuntos até chegar nas suas extensões, que são: Axioma da Escolha, Lema de Zorn, teorema da Boa ordenação, Espaços Vetoriais, Espaços Normados e Bases de Vetoriais, que não possuem seus conceitos totalmente definidos no ensino regular (Silva \& Jesus, 2007).

Existem equivalências em teorias dos conjuntos que são bem conhecidas como o axioma da escolha, lema de Zorn, teorema da boa ordem e o teorema de Tychonoff, todos esses são equivalentes, assim como existem aplicações utilizando o lema de Zorn na álgebra que são bem conhecidas, como a sua aplicação na base de Hammel, em Anéis, etc.

\section{Resultados e Discussão}

Nessa seção será demonstrada a equivalência do Axioma da Escolha e o Lema de Zorn, e uma aplicação do próprio lema na álgebra Linear. Ambos têm suas importâncias na teoria dos Conjuntos e em álgebra, o Axioma da Escolha é 
importante na construção axiomática dos conjuntos, mesmo não sendo importante nesta área de axiomatização, o lema de Zorn por sua vez, se demonstra importante não apenas na teoria dos conjuntos, mas também na área de análise funcional, topologia e álgebra como já foi dito antes.

Para a equivalência precisaremos de alguns conceitos básicos que serão vistos a seguir e, para a aplicação, será considerado que o leitor já possua alguns conhecimentos na área de álgebra linear, tais como as definições a seguir:

Axioma de Extensão: Dois conjuntos são semelhantes se, e somente se, tem os mesmos elementos.

Conjunto Parcialmente Ordenado: Uma ordem parcial em um conjunto E é uma relação R em E que obedece as seguintes relações: Reflexiva, isto é, ( $a, a) \in \mathrm{R}$ para cada $\mathrm{a} \in \mathrm{E}$; Anti simétrica, isto é, $(\mathrm{a}, \mathrm{b}) \in \mathrm{R}$ e (b, a) $\in \mathrm{R}$ implica que $\mathrm{a}=\mathrm{b}$; Transitiva, isto é, (a, b) $\in \mathrm{R}$ e (b, c) $\in \mathrm{R}$ e (a, c) $\in \mathrm{R}$ (Tricotomia).

Elemento Maximal: Seja X um conjunto parcialmente ordenado, então a é denominado elemento maximal em X, se para todo $\mathrm{x} \in \mathrm{X}$ existir um $\mathrm{a} \in \mathrm{X}$ tal que $\mathrm{a} \leq \mathrm{x} \Rightarrow \mathrm{x}=\mathrm{a}$.

Cadeia: $\mathrm{S}$ é uma cadeia se, para todos $\mathrm{y}, \mathrm{z} \in \mathrm{S}$ tivermos $\mathrm{y} \leq \mathrm{z}$ ou $\mathrm{z} \leq \mathrm{y}$.

Função Escolha: Seja $X$ um conjunto infinito, então f: $P(X)-\{\varnothing\} \rightarrow$ X é dita uma função escolha para o conjunto X, se $f(a)$ $\in \mathrm{X}$ para todo $\mathrm{a} \in \mathrm{P}(\mathrm{X})-\{\varnothing\}$.

Axioma da Escolha: Se I é um conjunto qualquer de índices e (xi) com i $\in$ I, é uma família de subconjuntos de um conjunto C tal que $x i \neq \emptyset$, para qualquer que seja $\mathrm{i} \in \mathrm{I}$, o produto $\prod i \in I$ de Xi é não vazio, HR (2001).

\subsection{Equivalência entre o Axioma da Escolha e o Lema de Zorn}

O axioma da escolha tem suas peculiaridades de afirmar que determinado conjunto existe (ou não existe), mas não dá nenhuma informação sobre como este conjunto pode ser construído e, por isso, foi rejeitado por diversos matemáticos que apresentaram resultados e formas equivalentes, aparentemente de mais fácil manuseio. Dentre estas formas encontram-se o Lema de Zorn.

\subsection{Demonstração da Equivalência}

Para iniciar a demonstração da equivalência, tomemos o Lema de Zorn como verdade, (Grace, 2010).

Seja $X$ um conjunto e $F$ um conjunto de funções de subconjuntos $P(X)$ em $X$, sendo $F=\{f: D \rightarrow X\}$, tal que o domínio de $\mathrm{f}=\mathrm{D} \in \mathrm{P}(\mathrm{X})$. Consideremos $\mathrm{A}$ um subconjunto de $\mathrm{D}$, e para todo $\mathrm{A} \in \mathrm{D} \Rightarrow \mathrm{f}(\mathrm{A}) \in \mathrm{A}$, assim a imagem de $\mathrm{f} \subset \mathrm{X}$ e como $\mathrm{P}(\mathrm{X}) \subset \mathrm{X}$ e o domínio de $\mathrm{f} \subset \mathrm{P}(\mathrm{X})$, temos que a imagem de f pertence ao conjunto do domínio $\mathrm{D}$, isto é, imagem de $\mathrm{f} \in$ D.

Suponhamos que F e D sejam parcialmente ordenados $(\leq)$, assim $\{\mathrm{f} 1 \leq \mathrm{f} 2 \leq \mathrm{f} 3 \leq \cdots \leq \mathrm{fn}\} \in \mathrm{F}$ e $\{\mathrm{d} 1 \subseteq \mathrm{d} 2 \subseteq \mathrm{d} 3 \subseteq \cdots$ $\subseteq \mathrm{dn}\} \in \mathrm{D}$, daí tomando d1 como o domínio de f1 e assim sucessivamente, se e somente se, $\{\mathrm{f} 1 \leq \mathrm{f} 2 \leq \mathrm{f}\} \leq \cdots \leq \mathrm{fn}\}$ e $\{\mathrm{d} 1 \subseteq$ $\mathrm{d} 2 \subseteq \mathrm{d} 3 \subseteq \cdots \subseteq \mathrm{dn}\}$, sendo assim, temos $\{\mathrm{f} 2 / \mathrm{d} 1=\mathrm{f} 1, \cdots, \mathrm{fn} / \mathrm{dn}-1=\mathrm{fn}-1\}$. Agora ordenando por extensão $\mathrm{F}$, temos que $\mathrm{f} 3 \subseteq$ f2 e assim por diante, como F e D são ordenados parcialmente $(\leq)$, claramente percebemos que $\mathrm{f} 1 \leq \mathrm{f} 1$ e d1 $\subseteq \mathrm{d} 1$ o que implica $\{\mathrm{f} 2 / \mathrm{d} 1=\mathrm{f} 1, \cdots, \mathrm{fn} / \mathrm{dn}-1=\mathrm{fn}-1\}$, pois é propriedade Reflexiva, sendo assim F e D possuem também a anti-simétrica $\mathrm{f} 3 \leq \mathrm{f} 4 \Rightarrow$ $\mathrm{f} 4 \leq \mathrm{f} 3$ e d $3 \subseteq \mathrm{d} 4 \Rightarrow \mathrm{d} 4 \subseteq \mathrm{d} 3$, o que os garante $\mathrm{d} 3=\mathrm{d} 4$ e $\mathrm{f} 3=\mathrm{f} 4$, podemos perceber que as funções de $\mathrm{F}$ podem ter o mesmo domínio, pela transitividade, temos $\mathrm{f} 3 \leq \mathrm{f} 4 \Rightarrow \mathrm{f} 4 \leq \mathrm{f} 5 \mathrm{e} \mathrm{d} 3 \subseteq \mathrm{d} 4 \Rightarrow \mathrm{d} 4 \subseteq \mathrm{d} 5, \operatorname{logo}\{\mathrm{f} 3 \mathrm{~d} 1=\mathrm{f} 1, \cdots, \mathrm{fn} \mathrm{d} 1=\mathrm{f} 1\}$ e assim, $F$ e $\mathrm{D}$ são ordenados.

Agora vamos provar que a validade do lema de Zorn implica no Axioma da Escolha. 
Tomando uma cadeia $Z$, tal que $Z=\{$ fi $\} \mathrm{i} \in \delta$, daí como $\mathrm{Z}$ é uma cadeia em $\mathrm{F}$, temos fi: $\mathrm{Di} \rightarrow \mathrm{X}$ e $\mathrm{Di} \in \mathrm{P}(\mathrm{X})$ como vimos no início dessa demonstração, e para qualquer que seja $\mathrm{A} \in \mathrm{Di}, \mathrm{f}(\mathrm{A}) \in \mathrm{A}$, pela ordem parcial $\mathrm{Dj}=\mathrm{U}_{\mathrm{i} \in \delta} D i$, podemos definir uma função f tal que fj: $\mathrm{Dj} \rightarrow \mathrm{X}$, pois $\mathrm{Di} \subseteq \mathrm{Dj}$ para todo $\mathrm{i}$, como $\mathrm{A} \in \mathrm{Di} \operatorname{logo} \mathrm{A} \in \mathrm{Dj}=\mathrm{U}_{\mathrm{i} \in \delta} D i$, daí deve existir um i0 em $\delta$ em que $A \in$ Di0 e pela $(\leq) \mathrm{fj}(\mathrm{A})=\mathrm{fi} 0(\mathrm{~A}) \in \mathrm{A}$ e fi0 $\leq$ fj para qualquer $\mathrm{i} \in \delta$, logo fj é uma limitante superior em $\mathrm{Z}$ e pelo lema de Zorn, existe fm em F que é o elemento maximal e o domínio de $\mathrm{fm} \subset \mathrm{P}(\mathrm{X})$. Sendo assim, suponhamos uma função escolha onde $\mathrm{Dm} \neq \mathrm{P}(\mathrm{X})-\{\varnothing\}$, assim Am deve pertencer a $\mathrm{P}(\mathrm{X})$ tal que $\mathrm{Am} \neq \mathrm{Dm}$, logo temos a função

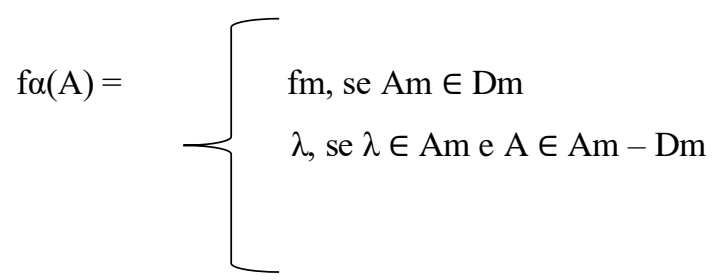

Definimos $\mathrm{D} \alpha=\mathrm{Dm} \cup\{\mathrm{Am}\}$ e $\mathrm{Dm} \subset \mathrm{D} \alpha$, a função escolha estende $\mathrm{A}$, a um único elemento se fm não for maximal, e se fm for maximal, então $A m \in D m$, como $D \alpha=D m \cup\{A m\}$, teríamos que $\mathrm{fm}<\mathrm{f} \alpha$, o que contradiz o lema de Zorn, sendo assim $\mathrm{Dm}=\mathrm{P}(\mathrm{X})-\{\varnothing\}$ e $\mathrm{F}$ 'e o conjunto de funções $\{\mathrm{f}: \mathrm{P}(\mathrm{X})-\{\varnothing\} \rightarrow \mathrm{X}\}$, tal que para todo $\mathrm{A} \in \mathrm{P}(\mathrm{X})-\{\varnothing\}$, teremos $\mathrm{f}(\mathrm{A}) \in$ A. Portanto, o Axioma de escolha e o Lema de Zorn são equivalentes.

\subsection{Aplicação do Lema de Zorn em Álgebra linear}

Uma das aplicações mais conhecidas do lema de Zorn se refere ao seu uso para facilitar a demonstração da existência, sob certas condições da base de Hammel.

\subsection{Base de Hammel}

Todo espaço vetorial admite uma base de (Hammel, 1972).

\section{Demonstração:}

Considerando B o conjunto, cuja seus elementos são subconjuntos de vetores LI de V. Podemos notar que B não é vazio, pois se $v \in V$, onde $V$ é o espaço vetorial, então $V$ é nulo pois o conjunto formado por $\{\mathrm{v}\}$ é LI.

Agora vamos considerar a relação de ordem dada pela inclusão de conjuntos de em $\mathrm{B}$, isto é, $\mathrm{C} \leq \mathrm{D}$, se $\mathrm{C} \subseteq \mathrm{D}$.

Suponhamos que $\{\mathrm{In}, \mathrm{n} \in \mathrm{N}\}$, seja uma cadeia em B, então UIn $\in \mathrm{B}$ pois dado um conjunto finito $\{\mathrm{e} 1, \cdots, \mathrm{en}\} \subset \mathrm{U}$ In, então existe $\mathrm{x} 0$ tal que $\{\mathrm{e} 1, \cdots, \mathrm{en}\} \subset \operatorname{In} 0$

Como In0 é formado por vetores LI, podemos concluir que F In é um conjunto de vetores LI, Pelo Lema de Zorn, existe um elemento em B que é o máximo In. Afirmamos que In é uma base de Hammel, efetivamente.

Dado e não pertencente a In, suponha que para qualquer combinação linear da forma

$$
\mathrm{ke}+\mathrm{k} 1 \mathrm{e} 1+\mathrm{k} 2 \mathrm{e} 2+\cdots+\mathrm{knen}=0 .
$$

Como $\{\mathrm{e} 1, \cdots, \mathrm{en}\} \subset \mathrm{Im}$, teremos $\mathrm{k}=0$. Então como $\{\mathrm{e} 1 \ldots$, en $\}$ são vetores $\mathrm{Li}$, teremos $\mathrm{k}=\mathrm{ki}=0$, onde $\mathrm{i} \in\{1, \cdots$, $\mathrm{n}\}$, e consequentemente In $\mathrm{U}\{\mathrm{e}\}$ seria LI. O que contradiz o fato de In ser elemento maximal. Portanto existe $\{\mathrm{e} 1 \cdots$, en $\} \in$ In e $\mathrm{k}, \mathrm{k} 1, \cdots, \mathrm{kn}$ com $\mathrm{k} \neq 0$, tal que

$$
\mathrm{ke}+\mathrm{k} 1 \mathrm{e} 1+\mathrm{k} 2 \mathrm{e} 2+\cdots+\mathrm{kn} \text { en }=0 .
$$

Temos

$$
\mathrm{e}=\frac{-k 1}{k} e 1-\cdots-\frac{-k n}{k} e n .
$$

Isso mostra que todo elemento de e de $\mathrm{V}$ pode ser escrito como combinação linear de 
Elementos de In (do máximo), sendo assim teremos sempre uma base de Hammel para cada espaço vetorial. Essa é uma aplicação bem clássica do Lema de Zorn em Álgebra Linear.

Essa é uma aplicação bem clássica do Lema de Zorn em Álgebra Linear.

\section{Conclusão}

A matemática tem mistérios inimagináveis, alguns teoremas ou lemas levam os nomes de seus supostos autores, mas o que não sabemos é que nem todos tem o nome de seu verdadeiro autor. Veja por exemplo o teorema de Pitágoras, muitos acreditam não ter sido Pitágoras que elaborou tal resultado e aqui temos o Lema de Zorn, que é um lema importante na área matemática. Portanto, conseguimos demonstrar a importância do Lema de Zorn e sua equivalência com o Axioma da Escolha, que torna o Lema de Zorn tão notório para os Estudiosos Matemáticos e Cientistas. E esta foi apenas uma das várias formas de equivalência que existem, pois temos na matemática outras equivalências e aplicações importantes.

Seguindo esta perspectiva, em trabalhos futuros, o lema de Zorn poder vir a ser aplicado na teoria dos Grafos e suas interconexões com a movimentação na Região Amazônica.

\section{Referências}

Beth, E (1964) The Foundations of Mathematics: A Study in the Philosophy of Science rev. ed. Harper Torchbooks.

Boccato, V. R. C. (2006) Metodologia da pesquisa bibliográfica na área odontológica e o artigo científico como forma de comunicação. Rev. Odontol. Univ. Cidade São Paulo, 18(3).

Celestino, R. (2000). Ensino-Aprendizagem da Álgebra linear: As pesquisas brasileiras na década de 90. (Dissertação de Mestrado, PUC-SP)

Campbell, P. J. (1978). The origin of Zorn's Lemma.Historia Mathematica. 77 (85), 77-89.

Cuesta, N 1955 Injusta atribucidn a Zorn de1 principio maximal Gac. Mat. (Madrid) (1) 7, 174-176 (MR 17, 931)

Diniz, M (2019). Teoria dos conjuntos: Sim ou Não? http://mathema.com.br/reflexoes/teoria-dos-conjuntos-sim-ou-nao-2/.

Fraenkel, A. A. \& Bar-Hillel, Y. (1958) Foundations of Set Theory Amsterdam (North-Holland) (MR 21, \#648)

Grace, A. K. S. G. S. (2010). Infinitos, Contínuos e Escolha: Teoria dos Conjuntos. (Dissertação de Conclusão de Curso, Universidade Federal de São Carlos)

Halmos, P. R. H. R. (2001). Teoria Ingênua dos Conjuntos. Editora Ciência Moderna.

Jech, T. J. (1973). The Axiom of Choice Amsterdam (North- Holland)

Lipschutz, S (1972). Teoria dos Conjuntos. McGraw-Hill Ltda.

Pereira, A. C. C.\& Saito, F. (2018). Os instrumentos matemáticos na interface entre história e ensino de matemática: compreendendo o cenário nacional nos últimos 10 anos. Boletim Cearense de Educação e História da Matemática, 5(14).

Rubin, H \& Rubin, J. R. H. (1963). Equivalents of the Axiom of choice. Noth-Holland Publishing company-Amsterdam.

Semadeni, Z. (1968) Notes to J. Mikusifiski's article "On Zorn's theorem" (Polish) Wiadom. Mat. (2) 10, 145-146 (MR 39, \#1273) (Passages quoted were translated by the author, with the assistance of Ann E. Lynch)

Silva, S. G., \& Jesus, J. P. C. (2007). Cem anos do axioma de escolha. Revista Matemática Universitária n॰42.

Suppes, P. (1960) Axiomatic Set Theory Princeton (Van Nostrand) 